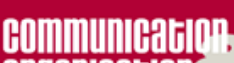

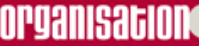

\section{Communication et organisation}

Revue scientifique francophone en Communication organisationnelle

$52 \mid 2017$

Communauté, sociabilité et bien commun: approche internationale

\title{
Société et Communauté : le tracé des frontières et l'idée du commun
}

Society and Community: The line of the borders and the idea of the "common" Sociedad y Comunidad: el trazado de las fronteras y la idea de lo común Sociedade e Comunidade : o traçado das fronteiras e a ideia do comum

Jean-Éric Douce

\section{(2) OpenEdition}

12 Journals

Édition électronique

URL : https://journals.openedition.org/communicationorganisation/5647

DOI : 10.4000/communicationorganisation.5647

ISBN : 979-10-300-0257-7

ISSN : $1775-3546$

Éditeur

Presses universitaires de Bordeaux

Édition imprimée

Date de publication : 1 décembre 2017

Pagination : 47-58

ISBN : 979-10-300-0163-1

ISSN : $1168-5549$

\section{Référence électronique}

Jean-Éric Douce, «Société et Communauté : le tracé des frontières et l'idée du commun »,

Communication et organisation [En ligne], 52 | 2017, mis en ligne le 01 décembre 2020, consulté le 02 janvier 2022. URL : http://journals.openedition.org/communicationorganisation/5647 ; DOI : https:// doi.org/10.4000/communicationorganisation.5647 


\title{
Sociēté et Communauté: Le tracé des frontiēres et l'idée du commun
}

\author{
Jean-Éric Douce ${ }^{1}$
}

«L'histoire des hommes est la longue succession des synonymes d'un même vocable ; y contredire est un devoir» René Char

Que sont nos sociétés devenues ? En Europe. En Occident. Et quelle rose des vents pour les guider ? Les levers de rideau en occident (là où le soleil se couche) sont souvent imprévisibles. Ainsi de notre millénaire qui ne correspond pas au film sur l'affiche. Quand nous pensions que la communauté (au sens de Tönnies, nous y reviendrons) laisserait la place à la société (avec ses loi et contrat) c'est bien au contraire la pratique communautaire qui, partout dans le monde, se substitue au contractualisme social et à l'universalisme citoyen. Comme toute histoire d'oiseau se termine par un chat, cette porte ouverte s'appelle le communautarisme. Ce qu'on croyait derrière nous est devant nous : enfance, cerveau reptilien, cocon et tribu. Une logique de régression. Cette thématique sera notre fil rouge.

Pour comprendre cela, il conviendra de revenir sur des distinctions fondamentales entre société, communauté et organisation. À suivre le tracé des frontières, cet état des lieux permettra d'abord de reconsidérer la problématique actuelle sur le «bien commun ». Que vaut cette notion, à quoi aspire-t-elle et s'oppose-t-elle ? Questions capitales. Ensuite, au-delà des définitions, nous reprendrons ce questionnement historique et originel : Qu'est-ce qui fait société ? Élaboration politique recommandée, donc, avec quelques opérations fondatrices et sans cesse renouvelées: Comment produire un singulier pluriel ? Un individu collectif ? Transformer les ils en nous et le $j e$ en nous? Bref il nous faudra renouer avec un problème névralgique (lieu

1 Docteur en philosophie et docteur en sociologie, consultant et formateur, chargé de cours et d'enseignement à Paris 1 Panthéon-Sorbonne et Paris 4 Sorbonne, auteur de publications sur l'histoire de la pensée, les besoins d’organisation sociale, l'Europe et le libéralisme politique ; Jean-Eric.Douce@univ-paris1.fr 
des douleurs) récurrent, celui qui consiste à fonder, réguler et équilibrer une société, une agglomération humaine, en termes de loi, de droit, de justice et d'égalité.

Nous vivons un temps de décollectivisation de l'action et de vide social, cette forme moderne de la pratique sociale qu'Hanna Arendt appelait " désolation $»^{2}$, « les sombres temps ».

Dans le monde de la concurrence généralisée, de la « déliaison ", celui de la marchandise, du système de l'équivalent général (ce que Marx appelait dans une jolie formule "les eaux glacées du calcul égoïste $»^{3}$ ), sur le théâtre de la scintillation marchande, il nous est demandé de rétablir des liens de solidarité et des espaces de socialisation. Notre cahier des charges a changé : à l'heure actuelle, les choses étant ce qu'elles sont, il ne s'agit plus de transformer le monde, noble tâche s'il en est, il s'agit de l'empêcher de partir en morceaux. Il est temps de produire des vues nouvelles sur l'au-delà de notre capitalisme financier, mondialisé et dérégulé, sans foi ni loi, qui n'est autre que le capitalisme $\mathrm{du} 19^{\mathrm{e}}$ siècle, de penser les formes et les conditions possibles de l'agir commun et de réfléchir conjointement aux formes et aux pratiques (organisationnelles et communicationnelles) d'une autre institution générale de la société. En conclusion nous proposerons donc une anabase, (cette épopée grecque du retour) soit une réorientation vers une destination difficile. Difficile, mais urgente.

Peut-on encore interroger F. Tönnies et sa présentation dichotomique des organisations sociales? Cette question devrait nous permettre de mieux comprendre quelques éléments cruciaux des évolutions contemporaines. Dans son ouvrage Communauté et Société ${ }^{4}$ Tönnies distingue deux types idéaux de structurations sociales : la Communauté et la Société. Précisons tout de suite que ces types idéaux fonctionnent d'une part comme tels, soit comme des abstractions exemplaires et que, d'autre part, ils correspondent à deux formes de la volonté humaine : la première, organique, concernant la Communauté et la seconde, réfléchie ou rationnelle, visant la Société . La Communauté, historiquement parlant, repose sur la famille, le plaisir, l'habitude et la mémoire, bref sur le sang, l'amitié et la foi6. La Société, quant à elle, et toujours historiquement parlant, repose sur la pensée réflexive et l'extériorisation de l'être, soit sur le contrat, la convention et l'échange ${ }^{7}$.

2 Condition de l'homme moderne, Ed. Calmann-Lévy, 1983.

3 Le manifeste communiste, Ed. La Pléiade, trad. M. Rubel et L. Evrard, 1963, tome 1, p. 157.

4 Communauté et société, Les catégories fondamentales de la sociologie pure, trad. et thèse complémentaire par Joseph Leige, PUF, 1946.

5 Livre 1, p. 1 sq. Et livre 2, p. 81 sq.

6 Ibid., il est question précisément de «sang, parenté, amitié, voisinage », p. 15.

7 Ibid., "La volonté commune dans chaque échange, dans la mesure où celui-ci est considéré comme un acte social, s'appelle un contrat », p. 43 et 45. 
Pour le dire autrement ${ }^{8} 1 \mathrm{a}$ Communauté figure un regroupement d'individus avec une langue, des intérêts, des buts et des biens partagés. Coutume et religion. Agrégat et frisson! La Société est de contrat et de loi qui fondent l'égalité de tous, autant dire une rencontre et un accord des volontés réfléchies sous le régime d'une législation politique.

À ce stade toutes les questions se posent et révèlent notre embarras. En tenant compte du point de vue historique de Tönnies, pertinent et efficace s'il n'est résolument original, la distinction Société/Communauté semble désormais peu opérationnelle. Et cela sur au moins deux points que nous prendrons comme hypothèses de travail. Le premier concerne précisément cette distinction qui fait suivre les wagons d'un train selon différents modes de régulation. Nous pensons que ces deux modes sont interactifs, activement corrélés, le sont ici et maintenant et le seront de plus en plus. Cette hypothèse, d'ailleurs, nous amènera à penser que dans notre société actuelle, usée, émiettée, fatiguée et liquide (Bauman 2006), la Communauté et les communautés servent et serviront toujours plus de refuges, abris confessionnels, familiaux et autres. Souvenons-nous également que l'idéal platonicien d'une communauté politique homogène à une « communauté de corps et d'âme »(La République), ce qui nous attend et ce que nous pouvons au demeurant souhaiter - la communauté européenne par exemple- n'est que l'aboutissement déguisé d'une déficience des normes et règles sociales frappées de caducité. Ainsi de la thèse d'Aristote qui critique l'unité totalisante préconisée par Platon et oppose le « vivre ensemble » au "vivre en commun ». Tönnies lui-même pointera cette confusion (de la Société et de la Communauté9), quand le droit familial de la communauté tend à se substituer au droit d'obligation, confusion que Durkheim, un peu plus tard, relèvera et entérinera.

Le deuxième point concerne la notion de «bien commun », ce pot au noir de la réflexion sociologique contemporaine. Tönnies encore fait remarquer que cette notion ne peut raisonnablement fonctionner dans une société - sauf à imaginer ou créer un sujet fictif ${ }^{10}$ - une belle intuition certes, mais bien entendu cette question demeure également pour le type communautaire. D'où, de nouveau une possible confusion entre Société et Communauté.

Plus avant il nous appartient de dire et de confirmer que le «Commun » n'est pas un bien ni un objet, mais une mobilisation (une pratique) politique d'acteurs sociaux dont la ou les volontés (Arendt 1983) ${ }^{11}$ consistent à organiser

8 Ibid., Introduction de 1935 intitulée « Normalité et moralité ».

9 Op. cit., « De même que la volonté organique individuelle développe la pensée pure et la volonté réfléchie elle-même qui tend ensuite à dissoudre celle-là et à se la subordonner, ainsi nous observons chez les peuples historiques, le processus de développement de la Société et des formes sociales de la volonté réfléchie, à partir des formes de vie et de volonté primitives et communautaires ", p. 187 et 229.

$10 \mathrm{Ibid}$. « (dans la société) il n'existe pas en réalité, ici, de bien commun. Un tel bien peut exister par une fiction des sujets ; celle-ci n'est cependant pas possible autrement que par la construction d'un sujet commun ayant une volonté imaginée, auquel doit se rapporter cette valeur commune », p. 40.

11 H. Arendt, op. cit., p. 231 sq. 
la vie sociale commune. $\mathrm{Ni}$ plus ni moins qu'une dynamique égalitaire et un processus de subjectivation ${ }^{12}$.

Le «bien commun » versus l'intérêt général, la volonté générale ou le service public ? Nous ne sommes pas loin de le penser. Mais quel est alors l'enjeu d'un tel recouvrement idéologique ${ }^{13}$ ?

De même que l'éthique (financière et entrepreneuriale) cache l'absence d'éthique, de même le "bien commun " cache l'absence de commun. Il en est ainsi de la logique du capital, du morcellement de nos sociétés, des tribus numériques - dans le village global - et de la communauté européenne. Liste non exhaustive bien entendu.

Incertitude ô mes délices,

Vous et moi nous nous en allons

Comme s'en vont les écrevisses,

A reculons, à reculons

Guillaume Apollinaire, Le Bestiaire, ou Cortège d'Orphée, 1911

La thèse que nous défendons ici tient à ceci que les différences entre Société et Communauté tendent à s'estomper et que les frontières se déplacent ${ }^{14}$. Comme Sparte sans muraille ni forteresse, la société des affects, liquide et narcissique, produit et reproduit des pratiques communautaires qui, elles-mêmes, engendrent le communautarisme, plusieurs types de communautarismes. S'enchaînent dès lors, indépendamment d'une adaptation/absorption plus forte aux temps nouveaux et changeants, des ruptures de contrat, d'une part, et, d'autre part, des fictions universalisantes qui divisent autant qu'elles rassemblent (les élus et les damnés) ${ }^{15}$. Les sociétés (civiles cf. Hobbes) disparaissent, les communautés restent.

\section{Tertium non datur}

C'est que la logique du temps mondial est contraignante. Reprenons quelques points.

On sait depuis Platon et le mythe de Protagoras ${ }^{16}$ que deux formes de temporalité sont à l'œuvre dans l'Histoire : le rapport des hommes aux choses, à la nature et le rapport de l'homme à l'homme. Alors que le premier rapport est cumulatif, ouvert et sans limites (la technique ou le feu prométhéen) le second

12 Pour prolonger ce point essentiel que nous ne pouvons qu'effleurer, voir Ugo Mattei, « Rendre inaliénables les biens communs » in Le Monde Diplomatique, déc. 2011, et Commun, essai sur la révolution au XXIe siècle de Pierre Dardot et Christian Laval, Ed. La Découverte, 2014, p. 137 sq.

13 Pour importante qu'elle soit, cette question ne peut être développée dans cette contribution. Signalons cependant ceci que les nouvelles figures du capitalisme et du consumérisme (consumant) sont ici à l’œuvre. Une actuelle publicité d'Opel est à cet égard emblématique qui se résume à ceci : « L'avenir appartient à tous »! 14 Régis Debray, bien entendu, dont nous reprenons beaucoup d'idées et d'expressions, depuis Critique de la raison politique, Ed. Gallimard, 1981, jusqu'à Eloge des frontières, Ed. Gallimard, 2010, en passant par Cours de médiologie générale, Ed. Gallimard, 1991.

15 Étienne Balibar, «L'universel ne rassemble pas, il divise », Entretien, Le Monde Idées, 09/02/2017.

16 In La République, Ed. Garnier Flammarion, 1966. 
est figé, clos et répétitif (l'histoire bégaie !). D'où les confusions possibles car l'espace n'est pas le temps ni la communication la transmission. Thématique simple, mais trop souvent délaissée quand s'inversent et se mélangent les positions des deux Jules (César ou Verne). Le rapport de l'homme à l'homme nous intéresse particulièrement car il produit une thématique politique et religieuse (relier, vivre ensemble) autant que symbolique (symbolein, mettre ensemble). Ainsi les progrès matériels et les rêves humains suivent des cours distincts... C'est un premier point. Le deuxième, sans rentrer dans le détail des changements techniques, nous enseigne que le thermostat de l'appartenance fait équilibre et correspondance entre ces deux séquences historiques (nature et histoire) qui nous englobent et nous identifient. C'est que le nouveau temps mondial, de flux et de dissémination sinon de chaos, impose de nouveaux schémas d'intégration et de socialisation. Le génie de la bouteille s'est déterritorialisé, externalisé, et si l' « homo faber » avance, l' " homo demens ", quant à lui, tourne en rond, voire recule. La mondialisation va de pair avec la tribalisation comme la fusion avec la fission, la globalisation avec le sécessionnisme et le satellite avec le clocher. Dit autrement, le système de l'équivalent général (monnaie et marchandise) et l'insurrection des identités culturelles forment un ensemble articulé. Tel est le big bang des transformations radicales qui s'opèrent dans notre nouvel espace mondialisé et marchandisé.

Dans ce monde de changements déroutants et non maîtrisés, les individus se regroupent autour de préférences ou d'identités primaires, religieuses, ethniques, territoriales etc. Et dans ce monde, il y a ceux qui vont y être et s'appartenir et ceux qui n'y seront pas et ne s'appartiendront pas (hommes, pays et continents). Retour à la communauté, donc, et déconnexion : les connectés et les autres (exclus et humiliés) qui cherchent (chercheront) à exclure ceux qui les ont exclus et à humilier ceux qui les ont humiliés. La mondialisation crée (va créer), en réaction contre elle-même, un langage de frustration qui va déboucher sur la recherche du refuge identitaire. Là où la mondialisation échoue (et elle échouera toujours), là où elle crée de la dysfonction (et il y en aura toujours) alors l'anti-mondialisation servira d'emblème et l'ethnicisation se renforcera.

Cette dialectique entre l'universel et le particulier, entre le global et le local, n'échappe à aucune histoire, d'aucun groupe et d'aucun pays, en occident comme en orient, dans les montagnes afghanes comme à Gaza.

À partir de ces quelques invariants anthropologiques (postulats philosociologiques) en rapport avec certaines règles de temporalité et avec l' « identité narrative » du groupe, il nous est possible de déterminer quelques lois d'organisation de toute communion humaine, des invariances. La première disposition permanente concerne ces questions : quelles sont les conditions dorganisation du collectif ? Comment vivre ensemble et s'organiser? Comment établir un site sur un terrain vague et faire souche, planter sa tente dans le désert? 
Très concrètement en traçant une ligne (à Rome et chez Rousseau) ${ }^{17}$, en séparant un dedans d'un dehors, les inclus des exclus, les athéniens des barbares, brefen séparant l'autorisé de l'interdit. Cette démarcation horizontale s'appelle le sacré (en latin sancire signifie délimiter, entourer, interdire). Est-il besoin d'ajouter que le limen (la frontière) est inviolable et que les enfants de Remus ont tous perdu la tête?

Et puis cette seconde loi d'organisation comme un invariant avec toutes ses variations : il n'y a pas de vie collective sans croyance, sans que le groupe ne se réfère à quelque chose, un mythe de fondation, un Livre, sacré et sacralisé, un événement légendaire, une fiction maîtresse, un Dieu unique (ou 30000 en Inde) etc. Cet invariant transhistorique s'appelle une transcendance, un amont symbolique, en quelque sorte un point absent et sublime, une origine et un horizon, une perspective sublime et grandiose que tout le monde regarde et attend ${ }^{18}$.

Pour comprendre le groupe humain en l'état et ses évolutions/ transformations historiques il nous faut absolument penser ensemble son horizontalité et sa verticalité, "ses contours et ses conteurs ${ }^{19}$. Il n'y a pas d'autonomie entre les deux, mais une corrélation originelle et complexe, rassembleuse et excluante, entre ce qui clôt à l'horizontal et ce qui ouvre à la verticale, entre la communication et la transmission. Ces repères-là, qui font lien avant que de faire sens, perdus ou frappés de caducité, obsolètes ou mal en point, le groupe (quel qu'il soit) devra inévitablement les remplacer. Ces appels d'air sont de plus en plus fréquents. Quand l'Idée républicaine devient le mol drapeau européen et la Société de contrat une Communauté de sang...

Ce que nous voulons dire également c'est que tous les groupes ou collectifs, à l'instar des individus, se racontent des histoires, une Histoire, pour ne pas voler en éclats. Cette histoire est un récit (story telling) ${ }^{20}$ avec un début et une fin et des chapitres au milieu. Ainsi deux grands récits structurent la scène occidentale : le grand récit monothéiste en provenance de Jérusalem, celui de la Loi, et le grand récit du Logos, en provenance d'Athènes, celui du savoir et de la raison. Notons d'ailleurs qu'ici encore, sur ces terres emblématiques, se rencontrent la communication et la transmission. Ces deux récits dont le principe fondateur et la dynamique consistent à établir la prévalence nécessaire de l'intelligibilité sur la pulsionnalité, de la raison (ou de la foi !) sur la pulsion, sont fatigués. Et à ce point fatigués et frappés d'obsolescence qu'ils sont en passe d’être remplacés et subrogés par un autre récit, celui du « divin marché » ${ }^{21}$.

17 Dans le Discours sur l'origine de l'inégalité, bien entendu. Mais nous n'oublierons pas Claude Lévi-Strauss. 18 Régis Debray, toujours. Notamment Les communions humaines, Pour en finir avec "la religion ", Ed. Fayard, 2005, où cette idée pourrait se résumer à ceci, souvent écrit : «Pas d'Inter sans Meta».

19 Idem. Voir également Madame H., Ed. Gallimard, 2015.

20 Voir notamment, sur ce sujet, Nicole d'Almeida, Les promesses de la communication, Ed. PUF, 2001.

21 Dany-Robert Dufour, L'individu qui vient... après le libéralisme, Ed. Denöel, 2011. D'autres récits sont en « renaissance ", n'en doutons pas, liés à une promesse politico-religieuse (!) mais ce n'est pas, ici-même, notre propos. 
Sans rentrer dans le détail qui déborderait notre propos, retenons ceci que l'idéal de la modernité (Idée platonicienne) peut (et doit) se résumer ainsi : un sujet, une histoire et un savoir qui donne du pouvoir. La rupture de la modernité ( $17^{\mathrm{e}}$ siècle) réserve à la théologie la solution du problème des rapports entre l'homme et Dieu (fonction fabulatrice, idée régulatrice, croyance nécessaire, illusion, opium voire amphétamine etc.) quand dans le même temps la dynamique sociale devient ce que les hommes en font (société et communauté) en réglant le problème de la connaissance de la nature et de la place de l'homme dans le monde. Ensuite se posera la question du contrat social entre les hommes, ce que nous nommons l'universelle contrainte du symbolique et de l'appartenance, du montage symbolique et de l'intégration. En quoi nous retrouvons nos propos liminaires : comment faire un singulier pluriel ? Et pour quelle religion civile (Rousseau) apte au rassemblement ?

En ces temps de glissement et de perte de sens, nous plaidons pour le retour du Politique, lato sensu, et l'advenue du Commun, stricto sensu. " Je ne te demande pas de m'aimer, je te parle de fraternité. Je te demande d'être à mes côtés pour la profanation du malheur » Romain Gary (Clair de femme).

$\mathrm{Ce}$ dernier point fait transition qui revient sur nos urgences. Si les frontières disparaissent (en l'état remplacées par des murs) ainsi que nos enclos rousseauistes (remplacés par des barbelés), si nos verticalités s'effritent et retombent en éclats confessionnels et sectaires, c'est alors que les principes communautaires reprennent de la force. Avec la famille, les ethnies, le sang et la foi... Ces préférences, bien entendu, sont aussi une façon de se tenir la main et d'avoir chaud.

Dans un récent article sur l'idée du commun J. L. Nancy ${ }^{22}$ 'emploie très efficacement à revisiter les termes de communisme et de commun. Si le mot communisme renvoie bien à une métaphysique (une ontologie du sujet -être c'est être avec), en ce lieu où nous retrouvons bien les différents sens de communauté, de communion et de communication, la notion de commun renvoie directement à l'idée de circulation et de partage, une ouverture et un espace entre étants. Cette ouverture et cet espace, qui ne clôturent pas le sujet ni ne le totalisent, impliquent son propre engagement dans la relation à l'autre. "C'est cela précisément qui est nommé parfois " amitié ", parfois " amour », ou « confiance », « fidélité », ou " dignité », parfois « art », parfois " pensée " parfois même "vie » ou " sens de la vie », dans tous ces noms il y a toujours, sous diverses modalités et tonalités, un engagement dans le Commun, pour lui et par lui $»^{23}$.

Certes ce point est essentiel, trop souvent oublié, mais nous nous permettrons d'y adjoindre un effet de réversion intrinsèque à cette dialectique de l'engagement. Janus étant notre cousin et Darwin notre maître en communication aléatoire et équivoque, si nous rajoutons la solidarité et la

22 In L'idée du communisme, sous la direction de A. Badiou et S. Zizek, Ed . Lignes, 2010.

23 Idem, p. 209-210. 
fraternité à cette liste rayonnante, nous nous apercevrons que toutes ces notions sont derechef des frontières contre le mal et la violence tout autant qu'elles peuvent susciter ou encourager ces mêmes productions de la haine et de l'intolérance.

Michelet nous avait pourtant prévenus (Journal-1847) : «La difficulté est celle-ci : 1 . Si la fraternité est laissée au sentiment, elle n'est pas efficace ou elle l'est pour une heure d'élan. 2. Si elle est écrite en Loi et impérative, elle n'est plus fraternelle. 3. Si vous voulez qu'elle s'étende, il faut quelle soit volontaire, et alors nous retournons au sentiment que nous avons quitté ». Et Platon déjà, vieillissant et désabusé, confirmait dans son dernier livre que, compte tenu de l'infirmité de la nature humaine, la Cité avait besoin de «Lois ». Des lois, faute de mieux... Les hommes ne sont pas des dieux.

Que le commun sépare, tout comme la communication, c'est une évidence puisque ni l'un ni l'autre n'englobent et ne font se rencontrer les formes variées et sans cesse représentées des conflits humains ${ }^{24}$. Ainsi en est-il également des notions d'hospitalité, d'accueil, reconnaissance et bienveillance qui peuvent aisément se transformer en leur contraire. Idem pour la fraternité (la « fraternité-terreur » disait Sartre, cette fièvre communautaire).

De même que la frontière est là pour éviter la guerre ou bien est le moyen de la faire, de même le commun est-il le pire et la meilleure des choses et le Politique, qui est gestion et reconnaissance des conflits pour éviter les guerres, les annonce-t-il souvent et les produit-il perpétuellement « N'est large espace que douleur et n'est univers que de sang, si loin que j'aille rien n'y change » Pablo Neruda.

Que faire et que nous est-il permis d'espérer, pour reprendre deux célèbres formules ? Comment faire monde commun ou bien établir une loi commune de lêtre ensemble?

En sachant déjà ce que les mots recouvrent et en distinguant bien la société de la communauté et des communautés, les glissements de sens, donc, pour ensuite réinventer ensemble, enfin, des temporalités et des spatialités aptes à l'expression des communions humaines. Deux exemples pour conclure. La première vignette concerne le principe société qui se réduit le plus souvent à des pratiques communautaires. Ce que produisent les nouvelles technologies de l'information et de la communication (parlons portables, tablettes et écrans) c'est une nouvelle actualité du fétichisme autrement dit un étrange mécanisme d'oblitération de la réalité (avec ses avantages et ses inconvénients) ${ }^{25}$. Ce dernier principe va de pair avec le pouvoir de la marchandise, soit la fétichisation de l'objet, la facilité des transactions, l'opacité des informations et l'effondrement

$24 \mathrm{Cf}$. le dialogue très instructif entre Jacques Derrida et Jurgen Habermas in Le «concept » du 11 septembre, Dialogues à New York (octobre-décembre 2001) avec Giovanna Borradori, Ed. Galilée, 2003.

25 Comme toutes les manifestations du fétichisme, cf. Jean-Eric Douce, « Fétichisme et aliénation, à propos des nouveaux objets transitionnels ", in Les nouveaux objets transitionnels, sous la direction de Daniel Marcelli, Ed. Erès, 2016. 
de l'idée de perspective. Ce dernier point nous paraît être un élément-clé de ce nouveau temps mondial qui est le nôtre. En effet l'idée de perspective, née à la Renaissance, a déserté nos champs politique, technique, artistique et idéologique. Tout comme, d'ailleurs, l'idée de salut, quand on ne peut se passer ni du sens ni de la promesse. Ni du désir de salut... Restent alors le pouvoir, l'aliénation et la servitude. Quant à nos nouveaux moyens de communication, toutes ces connexions qui ne font pas connivence, encore moins lien social, ils font partie de ce que Evgeny Morozov appelle "le mirage numérique ${ }^{26}$. Tout cet espace communicationnel fait fond sur ce que Marx a analysé en termes d'aliénation ou de réification quand l'homme n'est plus que « la carcasse du temps ${ }^{27}$. Il nous revient donc de dire que le sujet n'a plus de recul possible et que face au « discours du maître » qui lui est tenu (la formule est de Lacan qui exprime l'imposition des normes) il est happé, pris dans les filets, enveloppé. Ceci s'appelle le narcissisme ; un narcissisme généralisé quand il y a prévalence de l'être plein sur la représentation ${ }^{28}$, cette non mise en scène de l'altérité. La transcription en termes psychanalytiques donne ceci : il y a substitution de l'image, du monde iconique, à la parole. Nous aurions donc affaire à une économie du signe et non plus du signifiant.

Osons encore l'analogie suivante en rapport avec ce que nous avons vu précédemment : ce qui nous construit, individuellement et collectivement, c'est-à-dire ce qui nous permet d'organiser le chaos et d'ordonner le désordre, tient en deux opérations comme autant d'invariants historiques, un espace sacré et une transcendance. Il est une nécessité pour tout petit d'homme (et être social) de s'inscrire à la fois dans l'espace et le temps, dans la communication et la transmission, dans l'horizontalité et la verticalité. Le tracé des frontières, cette clôture à l'horizontale, fait toujours corrélation avec l'infini de la transcendance, cette perspective mystérieuse et lointaine qui ouvre, précisément, à la verticale. Toute transition avec le réel néchappe pas à ces deux conditions, de la limite et de la croyance. Or nos nouveaux objets transitionnels (et là réside notre analogie), comme l'alcool ou toute conduite addictive (liste non close, ouvrons nos tablettes !) mettent à mal ce continuum spatio-temporel. Nos nouvelles prothèses techniques, qui ne font plus transition, nous déconnectent du monde réel.

Avantage et inconvénient, écrivions-nous. Une grande liberté d'un côté, de plaisir, certes, et de jouissance ou de repos, de divertissement pascalien, mais une aliénation et une servitude de l'autre.

Notre deuxième vignette en forme de propos conclusif concerne la question de la solidarité. Solidaires et solitaires nous sommes, nous ne pouvons pas vivre ni tout à fait en commun ni tout à fait en solitude. Ce postulat anthropologique

26 Le mirage numérique, pour une politique du Bid Data, Paris, Les prairies ordinaires, 2015.

27 Manuscrits de 1844, Ed. Sociales, 1972, p. 57 sq.

28 Sur ce thème du fétichisme et du narcissisme, voir Pierre Legendre, Dieu au miroir. Étude sur l'institution des images, Paris, Ed. Fayard, 1994 ; notamment le premier chapitre sur l'aliénation constitutive du sujet, p. 35. 
est un fonds de commerce pour toutes les formes de communauté qui réchauffent, enthousiasment et régénèrent. Les soldats de la frontière ne sont jamais loin s'ils ne sont pas déjà les marqueurs et les lieutenants (tenant lieu) de la communauté. Entre hérodianisme et zélotisme (imitation et résistance cf. A. Toynbee) nous devons donc restaurer des principes de vie en commun ou de prise de corps qui soient universalisables, évolutifs et vérifiables (le modèle est connu) selon des critères exclusivement politiques, c'est-à-dire liés à l'intérêt général. La Nation française (une transcendance) a dissous « corps » et « communautés » pour s'inventer et s'inscrire dans le temps et la rationalité historique ${ }^{29}$.

Il nous est demandé également d'avoir et de mettre en avant une perspective non essentialiste de la communauté ${ }^{30}$. A contrario la perspective substantialiste, qui est la force et la faiblesse de la communauté, nous le répétons, représente un danger pour toute organisation sociale et politique viable, c'est-à-dire capable de " totaliser » (sur le mode hégélien) les différents particularismes individuels.

Construire, en fin de compte, un Nous qui ne soit pas un Sujet, ou qui ne prétend pas l'être. Tel est l'enjeu.

Le monde s'use, son modèle économico-social se défait et aggrave les inégalités. Dans ses interstices, nous pourrions dire dans ses appels d'air, le principe communautaire trouve sa force et sa puissance de ralliement.

Nous plaidons quant à nous pour le principe du Commun. C'est une exigence. Et un combat. Celui que la solidarité peut mener pour autant qu'on la considère non comme une vertu morale, mais bien comme une vertu politique dont la grande ambition tient à la régulation des égoïsmes. La solidarité dit la fonction d'appartenance. Elle implique le nécessaire passage du Je au Nous et la prise de conscience de l'intérêt du collectif. Certes, à tous égards, la solidarité, comme la fraternité, s'appuie sur une distinction entre le frère et l'étranger. Elle inclut donc elle exclut. Où nous retrouverions d'ailleurs, et logiquement, les fondements du feu communautaire. De te fabula narratur. Pour sortir de cette difficulté, il nous faudra donc définir la solidarité (la fraternité) comme un lien qui unit sous la Loi. Rousseau toujours, celui de la religion civile, c'est-à-dire dégagée des emprises cléricales, nous avait bien dit que la solidarité allait de pair (devrait aller de pair) avec la citoyenneté et donc postulait, inévitablement, l'égalité entre tous les membres de la société. Pour que les hommes ne dépendent ni des dieux ni des hommes, il faut qu'ils dépendent de la Loi.

La solidarité dit le partage des biens et des maux, cette dette contractée ensemble, ce contrat qui engage la responsabilité de chacun des participants.

29 Cf. Jean-Luc Chappey, Constellations : Radicalités irrationnelles in la revue Agone, n 61, 2017, p. 38 sq.

30 Cf. R. Esposito, Communauté : origine et destin de la communauté, Éd. Odile Jacob, 2000. 
Ce que nous appelons le commun, dans le principe d'une société politique, qui fait de l'égalité, du partage, du lien et de l'universel ses irrésistibles fondations.

L'homme obligeant l'homme est une question de priorité, de priorité théorique aussi, s'entend.

Alors reste et restera toujours cette promesse, la promesse impensée et impossible : que nous soyons capables de construire une fraternité sans armes, un Nous de temps de paix pour éviter le Nous de temps de guerre et percevoir encore, comme Prométhée, « et quoique soudés aux rochers du temps, les sons et le parfum des filles d'Océan ».

\section{BIBLIOGRAPHIE}

ALMEIDA N. D', 2001, Les promesses de la communication, Paris, PUF.

ARENDT H., 1983, Condition de l'homme moderne, Paris, Calmann-Lévy.

BADIOU A. et ZIZEK S. (sous la direction de) 2010, L’idée du communisme, Paris, Lignes.

BALIBAR E., L'universel ne rassemble pas, il divise, Entretien, Le Monde Idées, 09/02/2017.

BAUMAN Z., 2006, La vie liquide, Paris, Fayard/Pluriel.

CHAPPEY J-L., 2017, Constellations : Radicalités irrationnelles in la revue Agone $\mathrm{n}^{\circ} 61$.

DARDOT P. et LAVAL C., 2014, Commun, essai sur la révolution au XXI siècle, Paris, La Découverte.

DEBRAY R., 1981, Critique de la raison politique, Paris, Gallimard. 2010. Éloge des frontières, Paris, Gallimard, 1991. Cours de médiologie générale, Paris, Gallimard, 2005. Les communions humaines, Paris, Fayard, 2015. Madame H, Paris, Gallimard.

DERRIDA J. et HABERMAS J., 2003, Le « concept » du 11 septembre, Dialogues à New York (octobre-décembre 2001) avec Borradori G. Paris, Galilée.

DOUCE J.-E., 2016, Fétichisme et aliénation, à propos des nouveaux objets transitionnels in Les nouveaux objets transitionnels (sous la direction de Marcelli D.), Toulouse, Erès.

DUFOUR D.-R., 2011, L’individu qui vient... après le libéralisme, Paris, Denoël.

ESPOSITO R., 2000, Communauté : origine et destin de la communauté, Paris, Odile Jacob.

LEGENDRE P., 1994, Dieu au miroir. Étude sur l'institution des images, Paris, Fayard. LÉVI-STRAUSS C., 1955, Tristes tropiques, Paris, Plon.

MARX K., 1972, Manuscrits de 1844, Paris, Éditions sociales, 1963. Le manifeste communiste, Paris, La Pléiade.

MATTEI U., 2011, Rendre inaliénables les biens communs, in Le monde diplomatique, Paris.

MOROZOV E., 2015, Le mirage numérique, pour une politique du big data, Paris, Les prairies ordinaires. 
NANCY J-L., 2010, Communisme, le mot, in L'idée du communisme (sous la dir. De Badiou A. et Zizek S.) Paris, Lignes.

PLATON, 1966, La République, Paris, Garnier Flammarion.

ROUSSEAU J.-J., 1964, Discours sur l'origine et les fondements de l'inégalité, Paris, La Pléiade.

TÖNNIES F., 1946, Communauté et société, les catégories fondamentales de la sociologie, Paris, PUF.

TOYNBEE A., 1996, L’histoire, Paris, Payot et Rivages.

Résumé : Cette contribution a pour objectif de reprendre les aspects définitionnels de la Société et de la Communauté afin de les confronter aux différentes évolutions contemporaines et à un nouveau temps mondial. Analyse et actualité, ainsi, aboutiront à ce constat que le principe communautaire (préférences privées) tend, pour toutes sortes de raisons et de plus en plus, à se substituer au principe Société (préférences publiques). Parallèlement, la notion de «bien commun » tend également à remplacer la notion d'égalité ou celle du « commun ». Nous évoquerons enfin, au-delà de ces glissements de sens et de comportements, quelques pistes pour restaurer de manière urgente les réquisits historiques et désormais novateurs de la société de Contrat : Loi, citoyenneté, solidarité, espace et temps, limite et croyance.

Mots-clés : «bien commun », « commun », frontières, invariant anthropologique, transcendance, sacré, limite, croyance.

\begin{abstract}
This contribution has for objective to take up the definitional aspects of the Society and the Community and to confront them with the various contempory evolutions and a new world time. Analysis and current affairs will show that the Community principle (private preferences) tends, for any sorts of reasons and more and more, to substitute itselffor the Society principle (public preferences). At the same time, the notion of "common good" also tends to progressively replace the notion of equality or that of the "common". We shall thus evoke, beyond these shifts in meanings and behaviors, some ways to restore urgently the historical requisits and from now on innovative of the society of Contract: Law, citizenship, solidarity, space and time, limitation and belief.
\end{abstract}

Keywords: "Common good», "Common», Borders (frontiers), Anthropological invariant, Transcendence, The sacred, Limitation, Belief. 